\title{
Elektrodenmaterialien für Hochtemperatur-OFW-Elemente
}

\author{
Denny Richter ${ }^{1}$, Holger Fritze ${ }^{1}$, Sergey Sakharov ${ }^{2}$, Esko Forsén $^{3}$, Elena Mayer ${ }^{4}$, Leo Reindl ${ }^{4}$ \\ ${ }^{1}$ Technische Universität Clausthal, Institut für Energieforschung und Physikalische Technologien, \\ Am Stollen 19B, 37081 Goslar, Deutschland \\ ${ }^{2}$ FOMOS Materials, 16 Buzheninova street, Moskau, 107023, Russland \\ ${ }^{3}$ Danish Technological Institute, Centre for Microtechnology and Surface analysis, Gregersensvej, \\ 2630 Taastrup, Dänemark \\ ${ }^{4}$ Universität Freiburg, Institut für Mikrosystemtechnik, Georges-Koehler-Allee 103, \\ 79110 Freiburg, Deutschland
}

\begin{abstract}
Dargestellt werden metallische und keramische Elektrodenmaterialien für hochtemperaturtaugliche Oberflächenwellenelemente (OFW) sowie deren Einsatzgrenzen und die zum Ausfall führenden Degradationsmechanismen. Die getesteten Materialien umfassen Edelmetalle der Platingruppe wie Platin, Iridium, Palladium und Rhodium sowie keramische Materialien wie LSM (Lanthan-Strontium-Manganit). Bei Platin und Palladium kann die Degradation bei hohen Temperaturen vor allem auf Agglomerationsprozesse zurückgeführt werden. Platinbasierte OFW-Elemente können bei einer Temperatur von $800{ }^{\circ} \mathrm{C}$ für mehrere Stunden betrieben werden. Im Fall von Iridium-Elektroden ist die Einsatzfähigkeit in sauerstoffhaltigen Atmosphären vor allem durch eine bei etwa $650{ }^{\circ} \mathrm{C}$ einsetzende Oxidation begrenzt.
\end{abstract}

\section{Einleitung}

Piezoelektrische Einkristalle wie Langasit ermöglichen die Entwicklung von Oberflächenwellensensoren für den Einsatz bei hohen Temperaturen. Speziell die Möglichkeit, OFW-Sensoren drahtlos anzusprechen, erlaubt den Einsatz in Umgebungen und Systemen, die mit konventionellen Sensoren bisher kaum zugänglich Sind. Das Substratmaterial Langasit lässt sich mit Hilfe des piezoelektrischen Effektes praktisch bis zu seinem Schmelzpunkt bei $1470{ }^{\circ} \mathrm{C}$ zu Schwingungen anregen [1]. Demgegenüber stellt die Hochtemperaturstabilität der Elektroden und der Verbindungstechnik den begrenzenden Faktor dar. OFW-Elemente erfordern Elektroden, die nicht dicker als $150 \mathrm{~nm}$ sein sollten und eine gute Haftung am Substrat sowie eine hohe Leitfähigkeit aufweisen. Eine Langzeitstabilität bei $600{ }^{\circ} \mathrm{C}$ sowie eine Stabilität über mehrere Stunden bei Temperaturen oberhalb von $800^{\circ} \mathrm{C}$ werden für die geplanten Anwendungen, wie z. B. Teststände für Flugzeugturbinen, gefordert. In dieser Arbeit werden daher die Einsatzgrenzen potentieller Elektrodenmaterialien diskutiert, die Degradationsmechanismen untersucht und Optimierungsmöglichkeiten erarbeitet. Neben Metallen wie Iridium und Platin werden auch keramische Elektrodenmaterialien wie LSM untersucht, die bereits in Hochtemperaturbrennstoffzellen als Elektrodenmaterialien Anwendung finden [5].

Auf Grund des hohen Schmelzpunktes und der chemischen Beständigkeit sind Edelmetalle wie Platin, Rhodium, Palladium und Iridium im Allgemeinen gute Kandidaten für den Einsatz bei hohen Temperaturen. Dennoch sind auch diese Elemente Einschränkungen unterworfen, die eine Verwendung als Dünnschichtelektrode bei hohen Temperaturen beeinträchtigen. Edelmetalle weisen oft eine unzureichende Haftung auf oxydischen Substraten auf, was die Verwendung von dünnen Titan- oder Tantalzwischenschichten zur Verbesserung der Haftung notwendig macht [2].

Die Degradation der Edelmetallschichten selbst wird durch Abdampfen, Oxidation und Agglomeration dominiert. Auf Grund des niedrigen Dampfdruckes der meisten Edelmetalle schränkt das direkte Abdampfen des Metalls bei hohen Temperaturen die Stabilität nur geringfügig ein. In sauerstoffhaltigen Atmosphären kann es jedoch zur Oxidation der Metalloberflächen kommen. Die dabei entstehenden flüchtigen Oxide tragen zu einem stetigen Masseverlust der Elektroden bei [3]. Darüber hinaus stellt die Agglomeration gerade bei dünnen Metallschichten einen wesentlichen Degradationsfaktor dar [4].

Die Herstellung der Testschichten erfolgt mittels Laserpulsabscheidung (PLD - pulsed laser deposition) und Magnetronsputtern. Für die Strukturierung der OFW-Elemente wird die Nano-Imprint-Lithografie eingesetzt, 
die eine kostengünstige Herstellung von Strukturen mit hoher Präzision ermöglicht. Die entsprechenden Proben werden mit Hilfe des Elektronenstrahlverdampfens metallisiert. Die Untersuchung der Hochtemperatureigenschaften der Schichten erfolgt mittels Widerstandsmessungen in Abhängigkeit der Temperatur, Röntgendiffraktometrie (XRD) und Rasterelektronenmikroskopie (REM).

\section{Ergebnisse}

\subsection{Platinbasierte Elektroden}

Platin- und Platin/Rhodium(10\%)-Schichten werden in Verbindung mit $10 \mathrm{~nm}$ dicken Titanhaftschichten auf polierten Langasitsubstraten abgeschieden. Die sich anschließende Bestimmung des Widerstands erfolgt bei Temperaturen bis $1100^{\circ} \mathrm{C}$, wobei in Schritten von $100 \mathrm{~K}$ für jeweils 6 Stunden vorgegangen wird (Bild 1a).
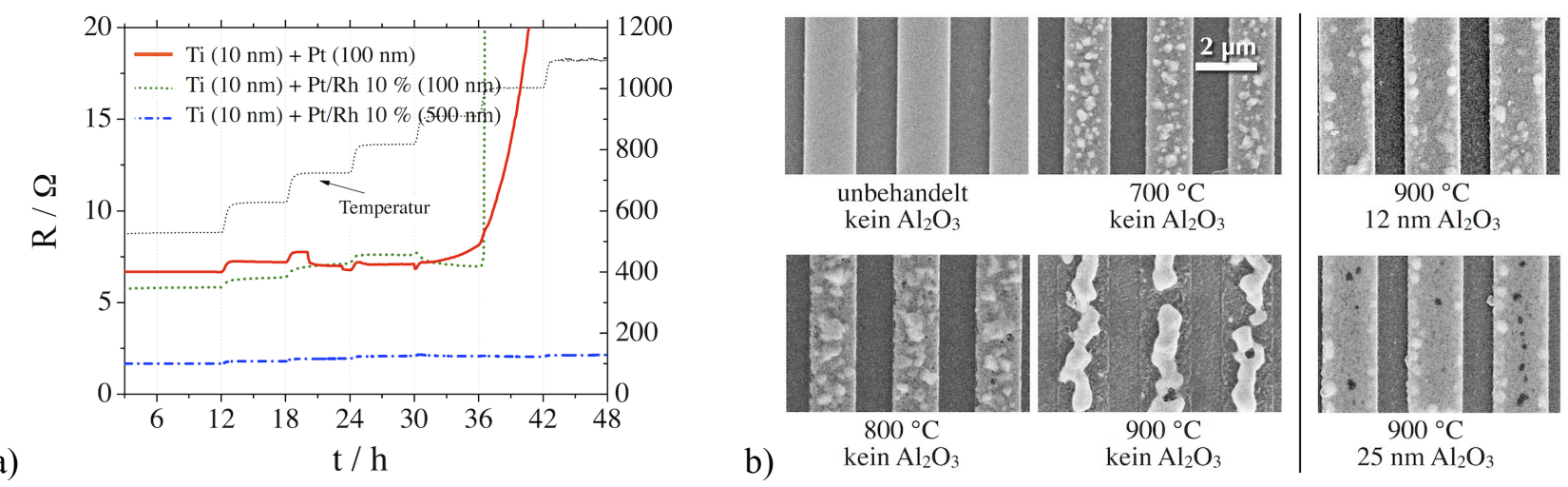

Bild 1 a) Temperatur und Zeitabhängigkeit des elektrischen Widerstands platinbasierter Schichten sowie b) REMBilder von Ti/Pt-Schichten $(10 \mathrm{~nm} \mathrm{Ti}+110 \mathrm{~nm} \mathrm{Pt})$ ohne und mit $\mathrm{Al}_{2} \mathrm{O}_{3}$-Schutzschicht nach Glühung bei verschiedenen Temperaturen.

Insgesamt $110 \mathrm{~nm}$ dicke Ti-Pt-Elektroden zeigen bis zu einer Temperatur von $800{ }^{\circ} \mathrm{C}$ stabile Widerstandswerte. Ab einer Temperatur von $900{ }^{\circ} \mathrm{C}$ ist ein starker Anstieg des Widerstands zu verzeichnen, der bei weiterer Erhöhung der Temperatur innerhalb weniger Stunden zu einer kompletten Zerstörung der Elektrode führt. Wie in Bild 1b dargestellt, wird die Zerstörung der Elektroden durch die zunehmende Agglomeration des Platins verursacht. Die Agglomeration kann durch das zusätzliche Aufbringen einer dünnen Aluminiumoxidschicht stark vermindert werden. Die Elektroden sind dann auch bei $900{ }^{\circ} \mathrm{C}$ einsetzbar. OFWElemente mit Ti-Pt-Elektroden lassen sich bis mindestens $800{ }^{\circ} \mathrm{C}$ für mehrere Stunden ohne Beeinträchtigung der Admittanz betreiben (Bild 2). Durch den Einsatz der beschriebenen Schutzschicht wird eine Erhöhung der Einsatzgrenze erwartet.

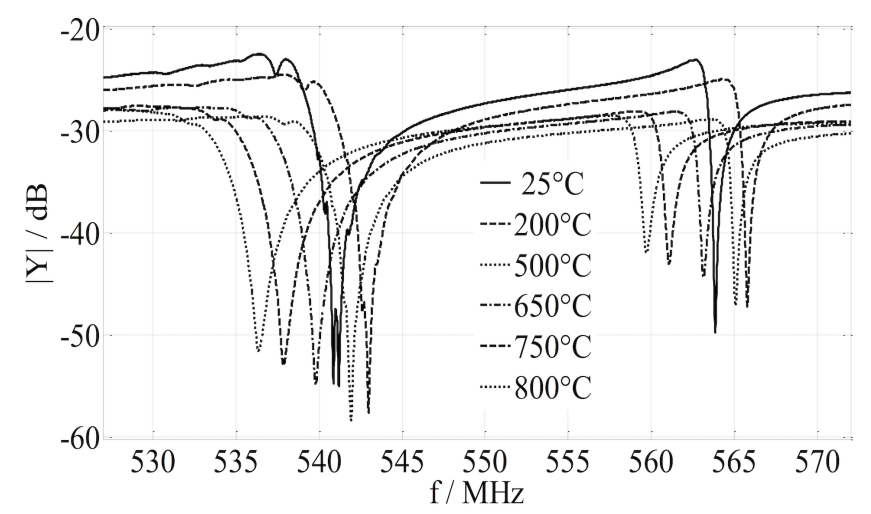

Bild 2 Admittanzspektren von Ti/Pt-beschichteten Langasit-OFW-Resonatoren bei Temperaturen bis zu $800{ }^{\circ} \mathrm{C}$. 
Eine weitere Möglichkeit zur Verbesserung der Elektrodenstabilität ist die Verwendung von PlatinRhodium-Legierungen an Stelle von reinem Platin. Diese Legierung weist einen höheren Schmelzpunkt und eine verbesserte Oxidationsbeständigkeit auf [6]. Eine Ti-Pt/Rh(10\%)-Schicht zeigt bei $900{ }^{\circ} \mathrm{C}$ eine im Vergleich zu reinen Ti-Pt-Elektroden erhöhte Stabilität. Bei Temperaturen von $1000{ }^{\circ} \mathrm{C}$ tritt aber auch hier eine Agglomeration auf, die zur Zerstörung der Schicht führt.

\subsection{Iridiumelektroden}

Weiterhin werden 100 bis $200 \mathrm{~nm}$ dicke Iridiumschichten auf Langasit abgeschieden. Im Vergleich zu den platinbasierten Elektroden zeigt sich auch ohne zusätzliche Haftschicht eine gute Haftung auf dem Substrat. Eine mögliche Erklärung für dieses Verhalten ist die Streckfestigkeit von Iridium, die im Vergleich zu anderen Elementen der Platingruppe etwa doppelt so groß ist $[7,8]$. Bis zu einer Temperatur von $650{ }^{\circ} \mathrm{C}$ zeigt Iridium eine sehr gute Stabilität. Nach einer 150-stündigen Glühung bei dieser Temperatur kann keine signifikante Degradation der Schichten festgestellt werden. Erst bei $700{ }^{\circ} \mathrm{C}$ kommt es zu einer stetigen Erhöhung des Widerstandes gefolgt von einer vollständigen Zerstörung der Schicht bei $800{ }^{\circ} \mathrm{C}$ (Bild 3a).

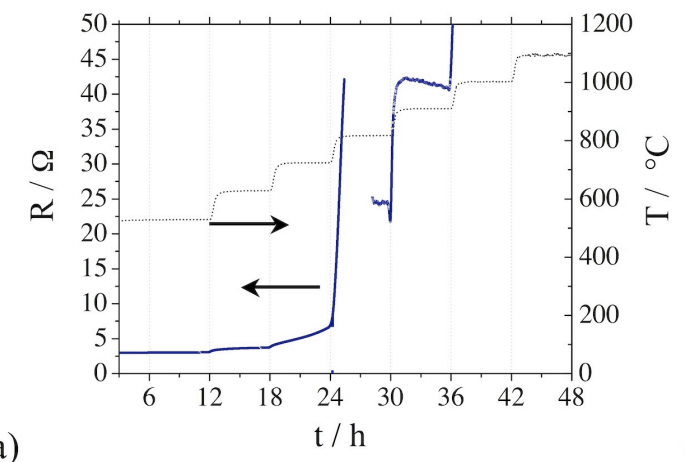

b)

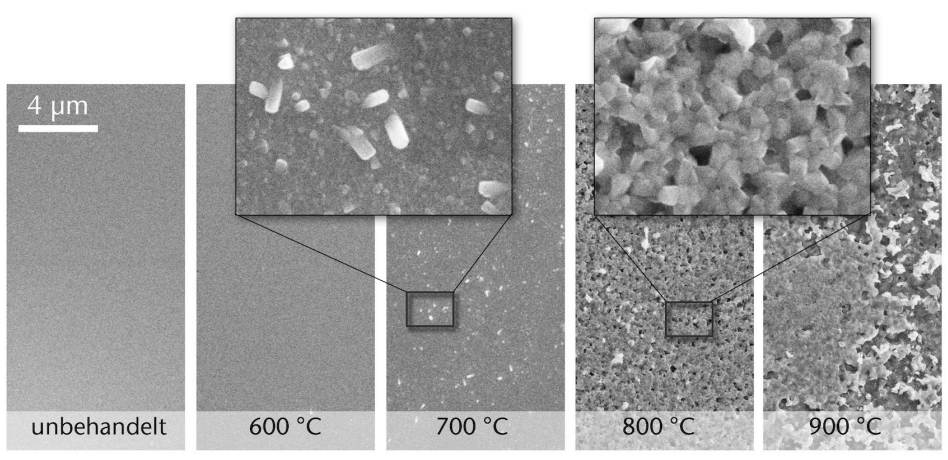

Bild 3 a) Temperatur und Zeitabhängigkeit des elektrischen Widerstands einer Iridium-Schicht und b) REMAufnahmen einer Iridiumschicht nach Glühung bei verschiedenen Temperaturen.

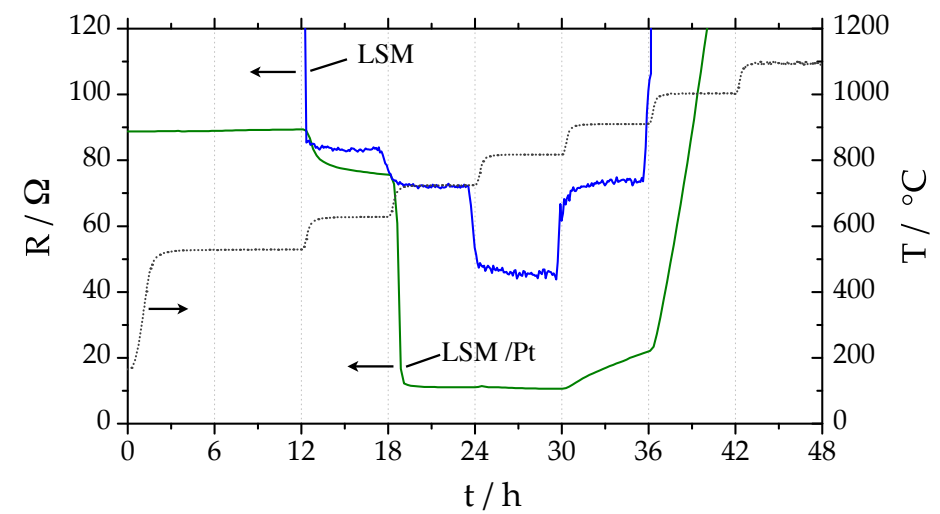

Bild 4 Temperatur und Zeitabhängigkeit des elektrischen Widerstands von LSM-basierten Schichten.

Die REM-Bilder zeigen, dass der Degradationsprozess nicht durch Agglomeration hervorgerufen wird. Die in Bild 3b hervorgehobenen kristallinen Strukturen können mittels XRD als $\mathrm{IrO}_{2}$ identifiziert werden, welches sich bei Temperaturen ab $700{ }^{\circ} \mathrm{C}$ bildet. Bei $800{ }^{\circ} \mathrm{C}$ wird ein kurzzeitiges Aussetzen des Widerstandssignals beobachtet, das durch den Übergang von $\mathrm{Ir} \mathrm{zu} \mathrm{IrO}_{2}$ verursacht werden könnte. Die danach gemessenen Widerstände sind etwa eine Größenordnung höher als zuvor, was in etwa dem zu erwartenden Widerstandswert von $\mathrm{IrO}_{2}$ entspricht [9]. Bei einer Temperatur von $1000{ }^{\circ} \mathrm{C}$ wird flüchtiges $\mathrm{Ir}_{2} \mathrm{O}_{3}$ gebildet, was zu einer völligen Auflösung der Elektrode führt. 


\subsection{LSM Elektroden}

Zusätzlich wird ein keramisches Elektrodenmaterial, LSM, abgeschieden. Die Widerstandsmessung (Bild 4) an einer $200 \mathrm{~nm}$ dicken Schicht zeigt bei einer Temperatur von $600{ }^{\circ} \mathrm{C}$ einen Wert, welcher um etwa den Faktor fünf bis zehn größer ist als der einer vergleichbaren Platinschicht. Bei Temperaturen unterhalb von $600{ }^{\circ} \mathrm{C}$ ist der Widerstand der LSM-Schichten so hoch, dass eine piezoelektrische Anregung kaum möglich ist. Um eine verbesserte Leitfähigkeit LSM-basierter Elektroden zu erreichen, wurden LSM und Platin im Volumenverhältnis 1:1 simultan mit Hilfe eines segmentierten PLD-Targets abgeschieden.

Die Dicke der hergestellten Schicht beträgt $100 \mathrm{~nm}$. Bei Temperaturen deutlich unterhalb von $600{ }^{\circ} \mathrm{C}$ konnte der Widerstand um den Faktor 20 im Vergleich zu reinem LSM verringert werden. Bei Temperaturen oberhalb von $600{ }^{\circ} \mathrm{C}$ ist der Widerstand nur um einen Faktor zwei höher als es bei reinen Platinelektroden zu erwarten wäre. Degradationserscheinungen zeigen sich ab einer Temperatur von $900{ }^{\circ} \mathrm{C}$. Durch eine Optimierung der Schichtzusammensetzung wird eine weitere Erhöhung der Stabilität und eine Verringerung der Widerstände erwartet.

\section{Zusammenfassung}

Bei einer Schichtdicke von etwa $100 \mathrm{~nm}$ zeigt $\mathrm{Pt} / \mathrm{Rh}(10 \%)$ die beste Stabilität unter den getesteten Metallen. Reine Platinschichten weisen eine Langzeitstabilität bis zu einer Temperatur von $700{ }^{\circ} \mathrm{C}$ auf. Entsprechende OFW-Elemente lassen sich im Bereich mehrerer Stunden auch bei $800{ }^{\circ} \mathrm{C}$ betreiben. Die Agglomeration, welche die Hauptursache der Degradation der platinbasierten Elektroden ist, lässt sich durch Aufbringen einer dünnen Aluminiumoxid-Schutzschicht vermindern und so die Stabilität der Elektroden signifikant erhöhen. Iridiumelektroden sind langzeitstabil bis zu einer Temperatur von $650{ }^{\circ} \mathrm{C}$. $\mathrm{Ab} 700{ }^{\circ} \mathrm{C} \mathrm{kommt} \mathrm{es} \mathrm{zu} \mathrm{einer}$ Degradation der Elektroden durch Oxidation. LSM/Pt-Elektroden zeigen im Vergleich zu reinen LSMElektroden eine verbesserte Leitfähigkeit und sind stabil bis zu einer Temperatur von $800{ }^{\circ} \mathrm{C}$.

\section{Danksagung}

Die dargestellten Arbeiten werden innerhalb des SAWHOT-Projektes, einem Gemeinschaftsprojekt europäischer und russischer Partner, durchgeführt. Die Forschungsarbeiten werden durch das Seventh Framework Programme ([FP7/2007-2013]) der Europäischen Union unter dem Grant Agreement n॰[NMP4- SL-2009247821] finanziell gefördert.

\section{Literatur}

[1] M. Schulz, D. Richter, H. Fritze, Material and resonator design dependant loss in langasite bulk acoustic wave resonators a thigh temperatures, in: Ultrasonics Symposium (IUS), 2009 IEEE International, IEEE, 2009, S. 1676-1679.

[2] G. Bernhardt, C. Silvestre, N. LeCursi, S. Moulzolf, D. Frankel, R. Lad. Performance of Zr and Ti adhesion layers for bonding of platinum metallization to sapphire substrates. Sensors and Actuators B: Chemical, 77 (1-2), 2001, S. 368-374.

[3] J. Chaston, Reactions of Oxygen with the Platinum Metals - Oxidation of Ruthenium, Rhodium, Iridium and Osmium, Platinum Metals Review 9 (2), 1965, S. 51-56.

[4] W. Maskell, N. Sammes, B. Steele, Agglomeration of thin platinum films on a zirconia substrate, Journal of Physics D: Applied Physics 20, 1987, S. 99-104.

[5] S.Lee, C.-L.Chu, M.-J. Tsai, J. Lee, High temperature oxidation behavior of interconnect coated with LSCF and LSM for solid oxide fuel cell by screen printing, Applied Surface Science 256 (6), 2010, S. $1817-1824$.

[6] H.Osamura, N.Abe, Development of new iridium alloy for spark plug electrodes, SAE transactions 108 (3), 1999, S. 1063-1074.

[7] K. Kreider and G. Gillen. High temperature materials for thin-film thermocouples on silicon wafers. Thin solid films 376 (1-2), 2000, S. 32-37.

[8] Platinum Group Metals Database, http://www.platinummetalsreview.com/jmpgm/

[9] M. A. El Khakani, M. Chaker, E. Gat, Pulsed laser deposition of highly conductive iridium oxide thin films, Applied Physics Letters 69, 1996, S. 2027-2029. 